\title{
Thermal Properties of the Frustrated Rb0.52(ND4)0.48D2PO4 System at Low Temperatures
}

\section{Authors: W.N. Lawless \& V. Hugo Schmidt}

This is a postprint of an article that originally appeared in Japanese Journal of Applied Physics in January 1985.

W.N. Lawless and V.H. Schmidt, "Thermal properties of the frustrated Rb0.52(ND4)0.48D2PO4 system at low temperatures," Japanese Journal of Applied Physics. 24, Suppl. 24-2, 952-954 (1985). http://dx.doi.org/10.7567/jjaps.24s2.952

Made available through Montana State University's $\underline{\text { ScholarWorks }}$ 


\title{
Thermal Properties of the Frustrated $\mathrm{Rb}_{0.52}\left(\mathrm{ND}_{4}\right)_{0.48} \mathrm{D}_{2} \mathrm{PO}_{4}$ System at Low Temperatures
}

\author{
W. N. LAwless and V. H. SCHMIDT* \\ CeramPhysics, Inc. Westerville, Ohio 43081 USA \\ *Department of Physics Montana State University Bozeman, Montana 59717 USA
}

\begin{abstract}
Specific heat and thermal conductivity data, 1.5-35 K, are reported on the mixed (frustrated) ferroelectric-antiferroelectric crystal $\mathrm{Rb}_{0.52}\left(\mathrm{ND}_{4}\right)_{0.48} \mathrm{D}_{2} \mathrm{PO}_{4}$. A glasslike linear term in the specific heat is resolved below $5 \mathrm{~K}$ and ascribed to random electric fields caused by the random distribution of $\mathrm{Rb}$ ions, in good agreement with the theory of McWhan et al. A maximum in $C / T^{3}$ at $13.5 \mathrm{~K}$ is attributed to dispersionless motions of the deuterium ions, by analogy with $\mathrm{KDP}$. In contrast to the specific heat, the thermal conductivity is not glasslike, displaying a maximum at $12.5 \mathrm{~K}$ of $37 \mathrm{~mW}$ $\mathrm{cm}^{-1} \mathrm{~K}^{-1}$. At lower temperatures, the behavior of the thermal conductivity is tentatively ascribed to resonant phonon scattering from the $\mathrm{Rb}-\mathrm{ND}_{4}$ system. The Debye temperature for the crystal is $303 \mathrm{~K}$, in reasonably good agreement with extrapolations based on $\theta_{\mathrm{D}}$ for KDP.
\end{abstract}

\section{§1. Introduction}

The crystal system $\mathrm{Rb}_{1-x}\left(\mathrm{NH}_{4}\right)_{x} \mathrm{H}_{2} \mathrm{PO}_{4}$ represents a frustrated polar system. The two end members at $x=0$ and $x=1$ order ferroelectrically and antiferroelectrically, respectively, and in the mixed system, $0.2<x<0.8$, no ordering occurs down to $9 \mathrm{~K} .{ }^{1)}$ Here the protons remained disordered as in ice, and Courtens ${ }^{2}$ has termed this a "proton-glass"' system by analogy with spin glasses.

Dielectric constant data $(\varepsilon)$ on these mixed crystals have been measured below room temperature (at $x=0.35^{3)}$ and $0.6^{4)}$ and for a $71.4 \%$ deuterated crystal at $\left.x=0.48^{5)}\right)$, and $\varepsilon$ displays a broad maximum $\left(\varepsilon_{\max } \sim 100\right)$ at about $30 \mathrm{~K}$ ( $70 \mathrm{~K}$ for the deuterated crystal). Schmidt et $a l .^{5)}$ have explained this unusual dielectric behavior using a model based on noninteracting asymmetric hydrogen bonds.

The disorder in these mixed crystals might be expected to impart glasslike thermal properties at lowtemperatures by analogy with the disordered cations on the conduction plane in the $\beta$-aluminas ${ }^{6)}$ and the disordered oxygen vacancies in stabilized zirconia. ${ }^{7)}$ The purpose of this paper is to report low-temperature specific heat and thermal conductivity measurements on a $71.4 \%$ deuterated crystal at $x=0.48$ to examine these expectations.

\section{§2. Experimental Methods and Results}

A twin-free single crystal in the form of a bar $\left(0.4 \times 0.6 \times 2.5 \mathrm{~cm}^{3}\right)$ was first used for thermal conductivity measurements, following which the crystal was cut in half to prepare a specific heat sample. Carbon-chip thermometers attached to the samples were calibrated in situ during the experiments, and all measurements were made in the adiabatic calorimeter described elsewhere. ${ }^{8)}$

Thermal conductivity data were measured by the oneheater, two-thermometer linear flow method wherein one end of the bar $(\mathrm{A} / \lambda=0.252 \mathrm{~cm})$ was fixtured with a $295 \Omega$ heater, and the other end was thermally anchored to a temperature-controlled reservoir. The largest experimental uncertainty comes from measuring the separation of the thermometers $( \pm 5 \%)$; however, the relative uncertainty between points is far less, $\sim \pm 1 \%$. The results of these measurements, $2-37 \mathrm{~K}$, are shown in Fig. 1.

After cutting the bar, the two halves $(1.44 \mathrm{G})$ were varnished together with G. E. 7031, and a heater $(313 \Omega)$ and thermometer $(12 \mathrm{mg})$ were fixtured onto the sample. The sample was suspended from a long-time-constant thermal link $(\tau \sim 100 \mathrm{~s}$ at $2 \mathrm{~K})$ within the adiabatic shield of the calorimeter and specific heat data were measured by the dynamic pulse method. ${ }^{8)}$ The addenda constituted $3.6 \mathrm{wt} \%$ and contributed $13-4 \%$ to the total heat capacity between $1.7-32 \mathrm{~K}$, respectively. The uncertainty in the method is $< \pm 2 \%$. The results of these specific heat measurements are shown in Fig. 2 (laballed DRADP), and data for KDP are shown for comparison. ${ }^{8)}$

The data in Fig. 2 are plotted as $C / T^{3}$ to illustrate the two non-Cebye features for both DRADP and KDP: (1) Maxima in $C / T^{3}$ around $15 \mathrm{~K}$; and (2) Rapid rise in $C / T^{3}$ with decreasing temperature below $5 \mathrm{~K}$.

\section{§3. Data Analyses}

\subsection{Thermal Conductivity}

The thermal conductivity data $(\kappa)$ in Fig. 1 display a maximum at $12.5 \mathrm{~K}$ with $\kappa_{\max }=37 \mathrm{~mW} \mathrm{~cm}^{-1} \mathrm{~K}^{-1}$, and below $4.5 \mathrm{~K}, \kappa=\mathrm{AT}^{\mathrm{m}}$ with $\mathrm{m}=1.31$ and $\mathrm{A}=3.01 \mathrm{~mW}$

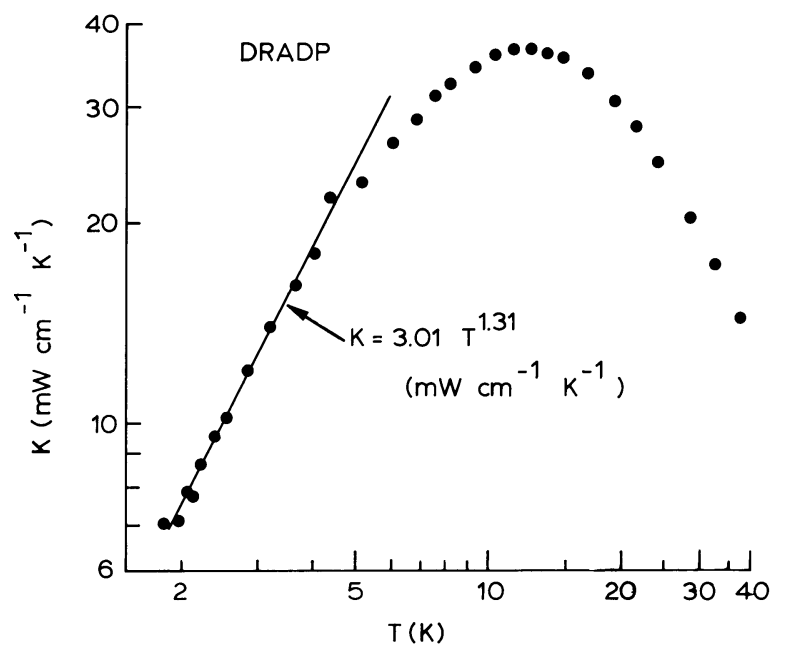

Fig. 1. Thermal conductivity data measured on the DRADP crystal. A maximum occurs at $12.5 \mathrm{~K}$, and below $5 \mathrm{~K}$ a $T^{1.31}$ behavior is seen. However, these data are not glasslike (see text). 


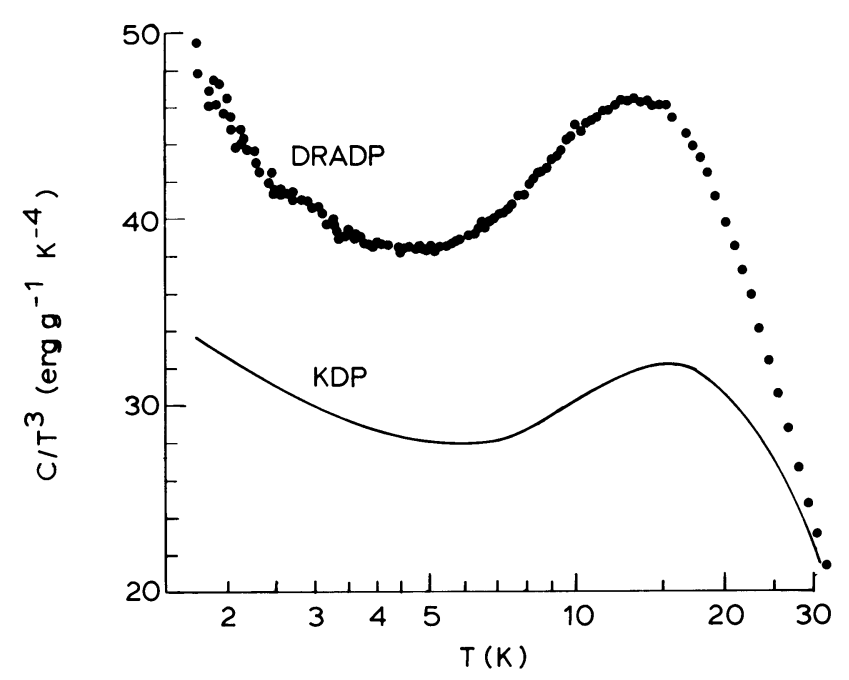

Fig. 2. Specific heat data for the DRADP crystal plotted as $C / T^{3}$. Data on KDP are shown for comparison.

$\mathrm{cm}^{-1} \mathrm{~K}^{-1}$ (least-squares fit). Although the temperature exponent $\mathrm{m}$ is in the glasslike range (so-called super-linear exponent), the $\kappa$-data in Fig. 1 do not reflect glasslike behavior for two important reasons: (1) Glasses have a plateau in $\kappa$ rather than a maximum; and (2) The $\kappa$-data for DRADP are about 30 times larger than typical glass data (e.g., the plateau in glasses occurs at $\kappa \sim 1 \mathrm{~mW}$ $\left.\mathrm{cm}^{-1} \mathrm{~K}^{-1}\right) .{ }^{9}$

\subsection{Specific Heat}

The most interesting feature of the specific heat data in Fig. 2 is the rapid rise in $C / T^{3}$ below $5 \mathrm{~K}$, and these data were analyzed according to

$$
C=m T^{3}+b T^{\mathrm{n}}
$$

where the $T^{3}$ term is the Debye background. Three cases were considered: (1) $n=1$, the glasslike term; (2) $n=3 / 2$, corresponding to the $T^{3 / 2}$ term seen in $\mathrm{KDP}^{10)}$; and (3) $n=-2$, corresponding to the Schottky term seen in some ferroelectrics. ${ }^{11)}$

The linear term yielded the superior least-squares fit to the experimental data, as shown in Fig. 3,

$$
C=36.35 T^{3}+36.43 T\left(\operatorname{erg~g}^{-1} \mathrm{~K}^{-1}\right) .
$$

The $T^{3 / 2}$ term in Eq. (1) yielded a marginally satisfactory fit, and the resulting $b$-coefficient was $\simeq 28 \mathrm{erg} \mathrm{g}^{-1} \mathrm{~K}^{-5 / 2}$. An additional reason for rejecting this $T^{3 / 2}$ fit is that this b-coefficient is far too large for the size crystal measured (compared to the data for small KDP crystals ${ }^{10)}$ ).

The Debye temperature for this DRADP crystal is determined from the m-coefficient in Eq. (2), $\theta_{\mathrm{D}}=302.9$ $\mathrm{K}$ (normalized to one atom per formula weight). We note in particular that the linear-term coefficient in Eq. (2) is $0.852 \times 10^{-5} \mathrm{~J} \mathrm{~cm}^{-3} \mathrm{~K}^{,-2}$ using a density of $2.34 \mathrm{~g}$ $\mathrm{cm}^{-3}$ and is in the glasslike range (i.e., $b \simeq 10^{-2} \mathrm{~J} \mathrm{~cm}^{-3}$ $\mathrm{K}^{-2}$ ).

The maximum in $C / T^{3}$ in Fig. 2 is certainly due to an Einstein-type mode(s), as found in all ferroelectric-type crystals. ${ }^{8,11)}$ A maximum in $C / T^{3}$ occurs at $T_{\max }=\theta_{\mathrm{E}} /$ 4.928 as an exact relation, where $\theta_{\mathrm{E}}$ is the Einstein temperature, provided a single einstein term is involved.

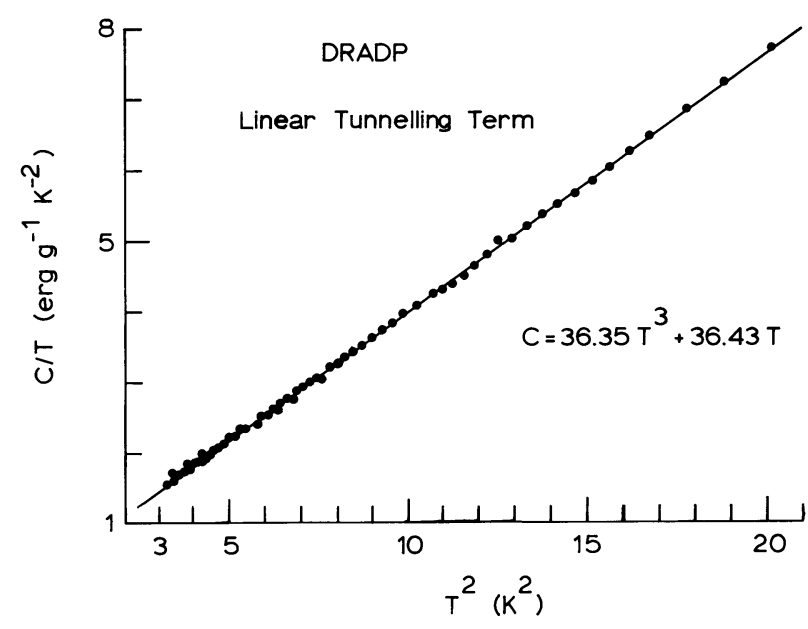

Fig. 3. Glasslike linear term in the specific heat of DRADP. The coefficient of the linear term is in the glasslike range and is in good agreement with the theory of McWhan et al. The fited Debye temperature is $303 \mathrm{~K}$.

Subtracting the linear term in Eq. (2) from the measured data, we find that $T_{\max }=13.5 \pm 0.51 \mathrm{~K}$, and, converting to frequency, $\omega_{\mathrm{E}}=46.24 \pm 1.71 \mathrm{~cm}^{-1}$.

For comparison, in KDP $\omega_{\mathrm{E}}=59.6 \mathrm{~cm}^{-1}$. ${ }^{8)}$ This leaves little doubt that the Einstein mode is due to the proton or deuterium motions since the ratio of these frequencies $(1.289 \pm 0.049)$ is very close to the ratio of the square root of the masses involved, $\mathrm{m}_{1} / \mathrm{m}_{2}=1.414$. Considerations of the reduced masses would bring this comparison into closer agreement.

\section{§4. Discussion}

These thermal measurements on DRADP show that a glasslike linear term appears in the low-temperature specific heat, but the thermal conductivity is more crystalline than glasslike.

A careful distinction has to be made regarding these thermal properties. In very general thermodynamic terms, the linear specific heat term requires only a constant density of (tunnelling) states in an insulator, whereas the glasslike $\kappa \propto T^{2}$ behavior also requires a two-level system that is localized (i.e., zero group velocity). ${ }^{12)}$ Therefore, a glasslike thermal conductivity does not necessarily accompany a glasslike specific heat.

The $\beta$-aluminas display a linear specific heat term, ${ }^{13)}$ and it is well known that these crystalline materials contain $\sim 30 \%$ excess cations on the conduction plane. Theoretical studies ${ }^{14)}$ of the disorder due to diffusing defects in a periodic potential have shown, however, that this does not led to the requisite linear term; rather, additional random electric fields are required. A probabilistic theory along this line has been given by McWhan et al., ${ }^{13)}$ according to which the $b$-coefficient in Eq. (1) is given by (for $n=1$ ),

$$
b=\left(\pi^{2} / 6\right) k^{2} P(0) x
$$

where

$$
P(0)=0.11\left[\left(e_{1}^{2} a / \varepsilon\right)\left(n_{1}+e_{2}^{3} n_{2} / e_{1}^{3}\right)^{2 / 3}\right]^{-1}
$$

Here $P(E)$ is the probability distribution that the energy difference between accessible sites is $E$, and $x$ is the 
number of accessible configurations per defect. In Eq. (4) $n_{1}, e_{1}$, and $n_{2}, e_{2}$ are the concentration and charge of the defects and compensating ions, respectively, $\varepsilon$ is the dielectric constant, and $a$ is the distance between accessible sites in the unit cell.

For the DRADP crystal, it is plausible to ascribe random electric fields to the $\mathrm{Rb}$ ions of concentration $n_{1}$, and adopting $a \simeq 4 \AA, \rho \simeq 2.43 \mathrm{~g} / \mathrm{cm}^{3}, \varepsilon=11.5,{ }^{5)} n_{2}=0$, and $x=1$ (normalized to one formula weight), Eqs. (3) and (4) yield $b \simeq 30 \mathrm{erg} \mathrm{g}^{-1} \mathrm{~K}^{-2}$, in remarkably good agreement with the experimental value $36.4 \mathrm{erg} \mathrm{g}^{-1} \mathrm{~K}^{-2}$, Eq. (2). Applying the same exercise to the disordered deuterium ions yields $b \sim 10^{3} \mathrm{erg} \mathrm{g}^{-1} \mathrm{~K}^{-2}$.

We therefore conclude that the linear term in the specific heat arises from the random distribution of $\mathrm{Rb}$ ions which in turn causes random electric fields. Going further, the consequent disorder does not play a major role in the thermal conductivity, due possibly to the nonlocalized nature of the $\mathrm{Rb}$ motions or to the formation of other than two-level tunnelling states. Specific heat data on crystals with varying $\mathrm{Rb}$ contents are needed to clarify these observations.

The unusual thermal conductivity data for the DRADP crystal, Fig. 1, is tentatively interpreted as follows: The maximum in $\kappa$ at $12.5 \mathrm{~K}$ is probably due to the onset of boundary scattering in the crystal (i.e., possibly due to microdomains within the crystal), but at lower temperatures the $\kappa \propto T^{3}$ boundary scattering limit is obscured by resonant phonon scattering from the $\mathrm{Rb}-$ $\mathrm{ND}_{4}$ ions, a situation similar to resonant scattering in doped alkali halides at low temperatures. Since the integrated scattering cross section depends on the density of $\mathrm{Rb}$ ions, measurements on crystals with varying $\mathrm{Rb}$ concentrations are needed to support these observations.

The picture that emerges from these studies is that the random distribution of $\mathrm{Rb}$ and $\mathrm{ND}_{4}$ ions in DRADP plays the major role in determining the low-temperature thermal properties of this crystal rather than the disordered deuterium ions which would otherwise be ordered ferroelectrically or antiferroelectrically in the end members. Both DRADP and KDP display maxima in $C / T^{3}$, and, as mentioned above, the Einstein frequencies involved with these maxima scale well with the masses of the proton and the deuterium ion. Going further, the Lindemann relation ${ }^{15)}$ predicts that $\theta_{\mathrm{D}} \propto M^{-5 / 6}$, other things being equal, where $M$ is the molecular weight, and using $\theta_{\mathrm{D}}=344 \mathrm{~K}$ for $\mathrm{KDP},{ }^{10}$ ) the relation predicts $\theta_{\mathrm{D}}=310 \mathrm{~K}$ for DRADP, in reasonably good agreement with the experimental value above, $303 \mathrm{~K}$. It is not clear why the $T^{3 / 2}$ term in the specific heat of $\mathrm{KDP}^{10 \text { ) }}$ is apparently missing in DRADP.

In conclusion, low-temperature thermal measurements on these frustrated polar-system crystals are a sensitive probe of the microscopic interactions involved and provide necessary information for a theoretical understanding of these new systems.

\section{References}

1) E. Courtens, T. F. Rosenbaum, S. E. Nagler and P. M. Horn: Phys. Rev. B209 (1984) 515.

2) E. Courtens: Phys. Rev. Lett. 52 (1984) 69.

3) E. Courtens: J. Phys. (Paris) Lett. 43 (1982) L199.

4) S. Iida and H. Terauchi: Proc. Autumn Mtg. Phys. Soc. Jpn. 2 (1983) 91.

5) V. H. Schmidt, S. Waplak, S. Hutton and P. Schnackenberg: Phys. Rev. B30 (1984) 2795.

6) P. J. Anthony and A. C. Anderson: Phpys. Rev. B14 (1976) 5198.

7) W. N. Lawless: Phys. Rev. B22 (1980) 3122; W. W. Lawless and T. K. Gupta: Phys. Rev. B28 (1983) 5507.

8) W. N. Lawless: Phys. Rev. B14 (1976) 134.

9) See the review by W. A. Phillips: J. Non-Cryst. Solids 31 (1978) 267.

10) W. N. Lawless: Phys. Rev. Lett. 36 (1976) 478; W. N. Lawless and T. D. Lawless: Ferroelectrics 45 (1982) 149.

11) W. N. Lawless: Phys. Rev. B19 (1979) 3755

12) W. A. Phillips, Amorphous solids, low-temperature properties (Springer-Verlag, Berlin, 1981).

13) D. B. McWhan, C. M. Varma, F. L. S. Hu and J. P. Remeika: Phys. Rev. B15 (1977) 553.

14) L. Pietronero, W. R. Schneider and S. Strässler: Phys. Rev. B15 (1977) 553.

15) E. S. R. Gopal, Specific heats at low temperatures (Plrnum, New York, 1960). 